Received: 13 March 2017

Accepted: 7 November 2017

Published online: 17 November 2017

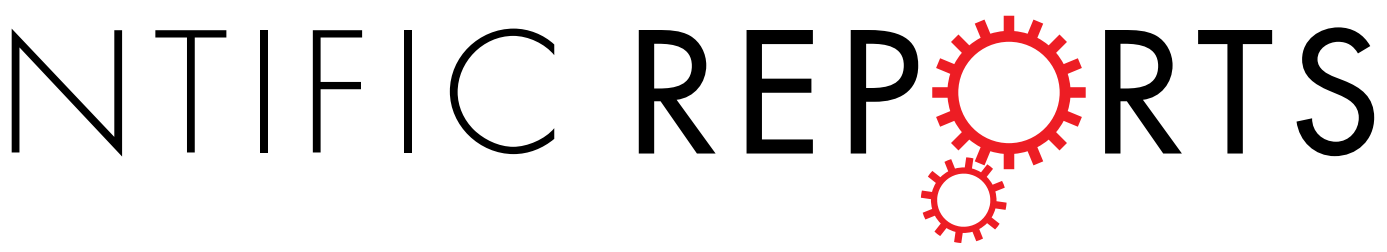

\title{
Global and Regional Effects of Bladder Cancer Risk Associated with Pioglitazone Therapy in Patients with Diabetes
}

Hua Qu ${ }^{1}$, Yi Zheng ${ }^{1}$, Yuren Wang ${ }^{1}$, Rui Zhang ${ }^{1}$, Xiongzhong Ruan ${ }^{2}$, Gangyi Yang ${ }^{3}$, Zhenqi Liu ${ }^{4}$ \& Hongting Zheng $\mathbb{1}^{1}$

It has been debated for several years as to whether the antidiabetic drug pioglitazone increases the risk for bladder cancer. A series of recent large population studies yielded conflicting results. To investigate why the observational studies yielded conflicting results, we conducted stratified analyses to analyze the potential confounders behind these discordant outcomes. A total of 2,764,731 participants from observational (OB) studies and 9,999 from randomized control trials (RCTs) were identified for these analyses. The stratified analysis revealed that the study type, adjustment for age/sex, treatment duration, cumulative dose, agents used in a control group, mean period of follow-up and study population region might contribute to the discordant outcomes. In terms of population regions, pioglitazone increased the risk for bladder cancer could be found in European population, and patients who undergo treatment with pioglitazone for longer durations ( $>12$ months) or are administrated a larger cumulative dose $(>28,000 \mathrm{mg})$ might require more attention, and the long-term effects $(\geq 3.6$ years) of pioglitazone needs be monitored more carefully.

Thiazolidinediones (TZDs), agonists of the peroxisome-proliferator-activated receptor (PPAR) $\gamma$, reduce blood glucose levels primarily by increasing insulin sensitivity in peripheral tissues, without causing hypoglycemia ${ }^{1,2}$. These agents have been widely used in patients with diabetes mellitus (DM). However, troglitazone was discontinued because it caused hepatotoxicity ${ }^{3}$, and the safety of rosiglitazone was disputed due its effect on the cardiovascular system $^{4,5}$. In contrast, pioglitazone has been shown to prevent the progression to diabetes and major cardiovascular events ${ }^{6,7}$ as well as nonalcoholic steatohepatitis ${ }^{6}$, thereby indicating a broader prospect for its clinical applications. However, globally, safety concerns pertaining to the potential effect of pioglitazone in increasing bladder cancer risk have been raised and debated for many years ${ }^{8-23}$. A series of large clinical studies ${ }^{24-26}$, such as the Kaiser Permanente Northern California (KPNC) study, United Kingdom Clinical Practice Research Datalink (UKCPRD) research, and Four European Countries Datasets (FECD) research, obtained discordant results, which led to more debate regarding this issue. Although this observation is still debated, the U.S. Food and Drug Administration (FDA) did warn about this risk on December 12, 2016 (http://www.fda.gov/Drugs/ DrugSafety/ucm519616.htm).

We conducted stratified analyses to investigate why the observational studies yield conflicting results and to analyze potential confounders that caused inconsistent results in the previous studies, as well as to determine optimal future study designs. In addition, as pioglitazone use may be related to other malignancies as well ${ }^{24,27}$, risk for other cancers types were also evaluated in the study.

\footnotetext{
${ }^{1}$ Department of Endocrinology, Xinqiao Hospital, Third Military Medical University, Chongqing, China. ${ }^{2}$ John Moorhead Research Laboratory, Royal Free and University College Medical School, University College London, Royal Free Campus, London, United Kingdom. ${ }^{3}$ Department of Endocrinology, the Second Affiliated Hospital, Chongqing Medical University, Chongqing, China. ${ }^{4}$ Division of Endocrinology and Metabolism, Department of Internal Medicine, University of Virginia Health System, Charlottesville, Virginia, USA. Hua Qu, Yi Zheng and Yuren Wang contributed equally to this work. Correspondence and requests for materials should be addressed to H.Z. (email: fnf7703@ hotmail.com)
} 


\begin{abstract}
Methods
Literature Search. Embase, PubMed, Web of Science, Cochrane Central Register of Controlled Trials (CENTRAL), and ClinicalTrials.gov were searched from inception through Jan 5, 2017 without language restriction. Two independent reviewers (Y.W. and R.Z.) searched and selected studies separately. Disagreements were resolved by discussion between the reviewers, and if necessary, consultation with other authors included in this study. Our search strategy included the following terms pertinent to pioglitazone: peroxisome proliferator activated receptor agonist/activator, PPAR, thiazolidinediones, TZDs, pioglitazone, Actos; and terms pertinent to cancer: cancer, tumor, carcinoma, neoplasm, malignancy (eAppendix 1 in the supplement). References of relevant studies were manually screened for eligible sources of data.
\end{abstract}

Inclusion and Exclusion Criteria. All human studies that evaluated patients with DM, reported pioglitazone therapy, and provided cancer outcomes were included. Observational (OB) studies and randomized controlled trials (RCTs) that provided either relative risk estimates such as risk ratios (RR), hazard ratios (HR) or odds ratios (OR) and 95\% confidence intervals (CI) for cancers or raw data were eligible. Studies with the greatest number of patients and the latest publications were selected when overlapping subjects were included in more than one study. Trials reporting serious adverse events or adverse events related to cancer following pioglitazone exposure were also included.

We excluded duplicate reports and abstracts from meeting proceedings. Studies were also excluded if they were animal research, reviews, comments or replies.

Data Extraction. Three reviewers (Y.W., H.Q. and R.Z.) independently extracted data from the primary texts and supplementary appendixes of all trials. Disagreements among the three reviewers were resolved by discussion, and if necessary, consultation with the other two reviewers (Y.Z. and H.Z.). The following data were collected for OB studies: authors, year of publication, age and sex, sample size, number of cancer events in both groups, RR, HR, OR with $95 \%$ CI, study type, adjustment factors, dose/duration response gradient, types of medications used in exposed and control groups, mean period of follow-up, target disease, and population region. For RCTs, data included trial registry number, number of study sites, study phase, number of cancer events in both groups, types of medications used in interventional and control groups, duration of follow-up, target disease, and population region.

For relative risk estimates of each study, we selected the most adjusted value (that is, the multivariable association measure with the highest number of covariates, to reduce the biases as much as possible) and corresponding 95\% CI, in addition to raw events data. Unadjusted estimates were selected if the outcome was not adjusted for any variable.

Quality Assessment. Three reviewers (Y.W., R.Z. and H.Q.) independently assessed the quality of all studies. The Newcastle-Ottawa quality assessment scale ${ }^{28}$ was used to assess the risk of bias of cohort studies and case-control studies. The highest-quality score was 9 (maximum), and studies with scores $\geq 7$ were considered as having a low risk of bias, scores of $4-6$ as having a moderate risk of bias, and scores $<4$ as having a high risk of bias. The item "was follow-up long enough for outcomes to occur" for cohort studies was removed owing to the adequate duration of follow-up is uncertain and was analyzed as a result of this study. The Cochrane Collaboration's tool ${ }^{29}$ was used to assess the risk of bias of RCTs. The judgmental items were "random sequence generation", "allocation concealment", "blinding of participants and personnel", "blinded assessment of bladder cancer events", "incomplete outcome data", and "selective reporting".

Definitions. The primary outcomes were included to examine the association between pioglitazone use and bladder cancer risk and whether this association varied based on the study design (i.e., study type, adjusted factors, intervention measures, comparator agents, follow-up duration, and study population $)^{30}$. The secondary outcome was defined as the relationship between pioglitazone use and other cancer risks.

Statistical Analysis. As the incidence rates of cancers involved in our study are relatively rare $(<5 \%)$ in overall and subgroup analyses, the distinctions among the RR, HR, and OR can be ignored ${ }^{31}$. We pooled relative risk estimates and reported pooled OR with corresponding 95\% CI using random effects models according to the methodology proposed by DerSimonian and Laird ${ }^{32}$, with weights calculated by the inverse variance method in cases of heterogeneity. Otherwise, fixed-effects models were used. Subgroup analyses were conducted to explore whether the association between pioglitazone use and bladder cancer risk were varied by study design.

The MOOSE guidelines ${ }^{33}$ for meta-analysis were followed, and PRISMA criteria ${ }^{34}$ were performed for reporting our meta-analysis. Publication bias was evaluated visually by funnel plots and quantified by the Egger's test and the Begg's test ${ }^{35,36}$. Heterogeneity across trials was assessed by the $I^{2}$ statistic, with values greater than $50 \%$ indicating significant statistical heterogeneity ${ }^{37}$. If significant statistical heterogeneity was detected, a sensitivity analysis was conducted using the "leave one out" approach ${ }^{38}$ to identify the source of heterogeneity. This "leave one out" approach was also used for sensitivity analysis to detect the influence of a single study on the overall bladder cancer risk. In addition, sensitivity analyses were also conducted by study quality analysis, which was restricted to the highest-quality studies with Newcastle-Ottawa scale scores of 8-9, and to test the robustness of overall bladder cancer risk and subgroup analysis.

Results with 2 -sided $P$-values less than 0.05 were considered statistically significant. All statistical analyses were performed using Stata Statistical Software: version 12.0 (STATA Corp, College Station, TX).

\title{
Results
}

Figure 1 outlines the procedure used for the literature search. For analysis of the primary outcomes (see Definitions in Methods), 19 OB studies ${ }^{9,12-22,24-27,39-41}$ and 4 RCTs (including clinical trials NCT00494312 and NCT00736099) ${ }^{10,42}$ published between 1986 and 2016 were identified. From the OB studies, 2,764,731 participants 


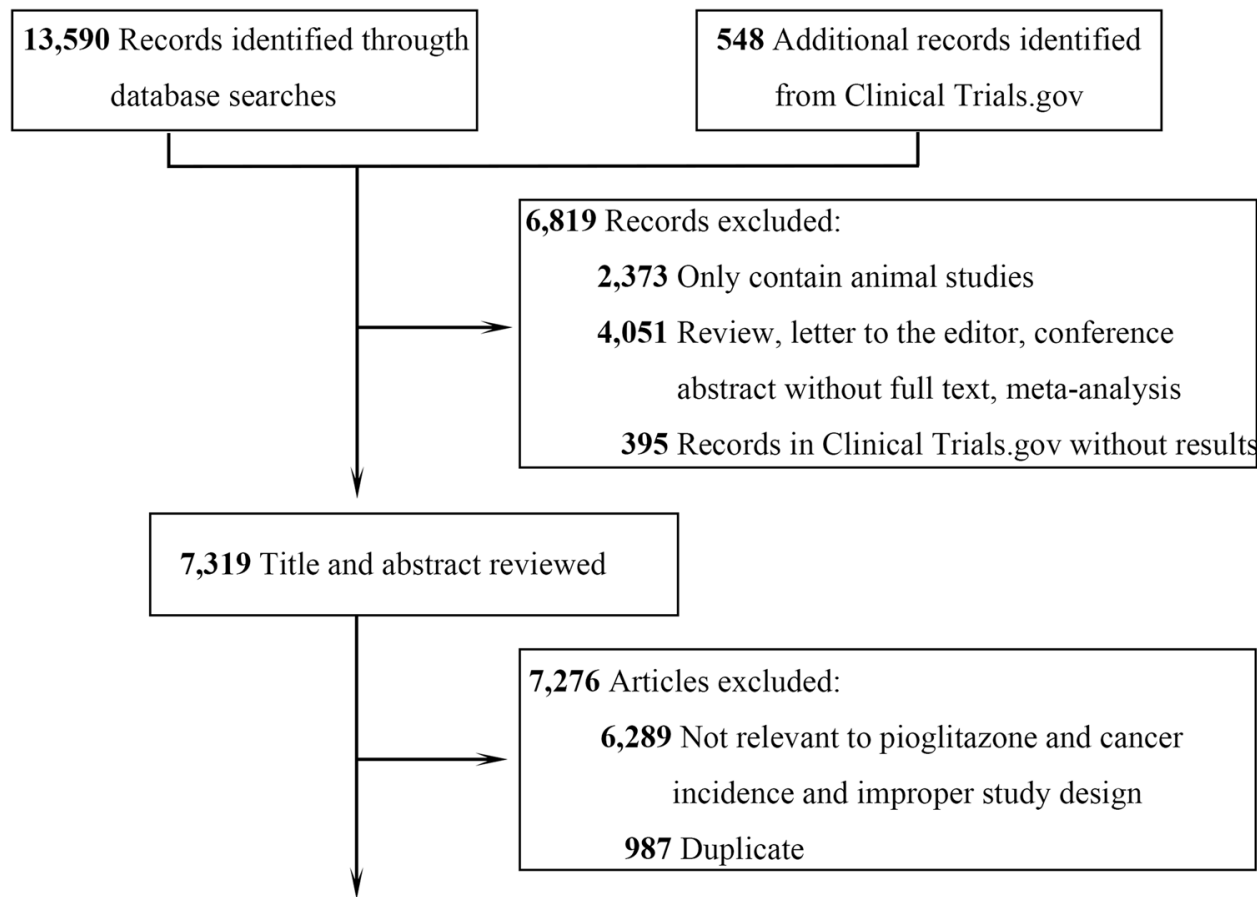

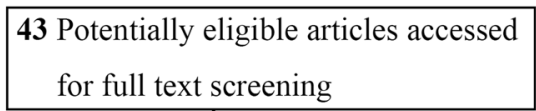

\begin{tabular}{|l|l|}
\hline & 10 Artiles excluded: \\
4 Data from same claims database \\
4 Inadequate data to calculate a risk estimate with \\
$95 \%$ confidence interval \\
2 Not patients with diabetes mellitus
\end{tabular}

2 Not patients with diabetes mellitus

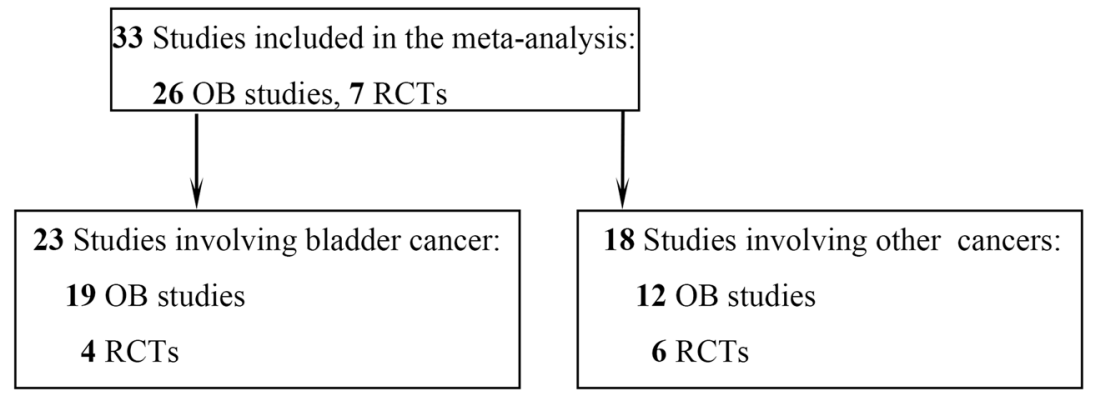

Figure 1. Flowchart of the Literature Search and Study Selection Abbreviations: OB, observational studies. RCTs, randomized controlled trials.

were included. Of these participants, 343,176 (12.4\%) were exposed to pioglitazone, and 13,264 (0.5\%) developed bladder cancer. The mean period of follow-up ranged from 2.1 to 7.9 years. In the RCTs, 9,999 participants were included. Of these participants, 4,515 (45.2\%) were exposed to pioglitazone, and $24(0.2 \%)$ developed bladder cancer. The mean period of follow-up ranged from 72 weeks to 48 months (eTable 1 and eReferences in the Supplement). For analysis of the secondary outcome (see Definitions in Methods), 12 OB studies 9,13,17,24,27,39,43-48 and 6 RCTs (including clinical trials NCT00736099, NCT00676338, NCT00879970, and NCT00637273) ${ }^{10,42}$ were identified, and 17 other site-specific cancers were assessed.

Part 1: Analysis with observational studies. Pioglitazone and overall bladder cancer risk at the global level. First, we conducted a pooled analysis to determine whether pioglitazone use was associated with the bladder cancer risk at the global level. To date, a majority of the studies related to this topic are OB studies. Only 


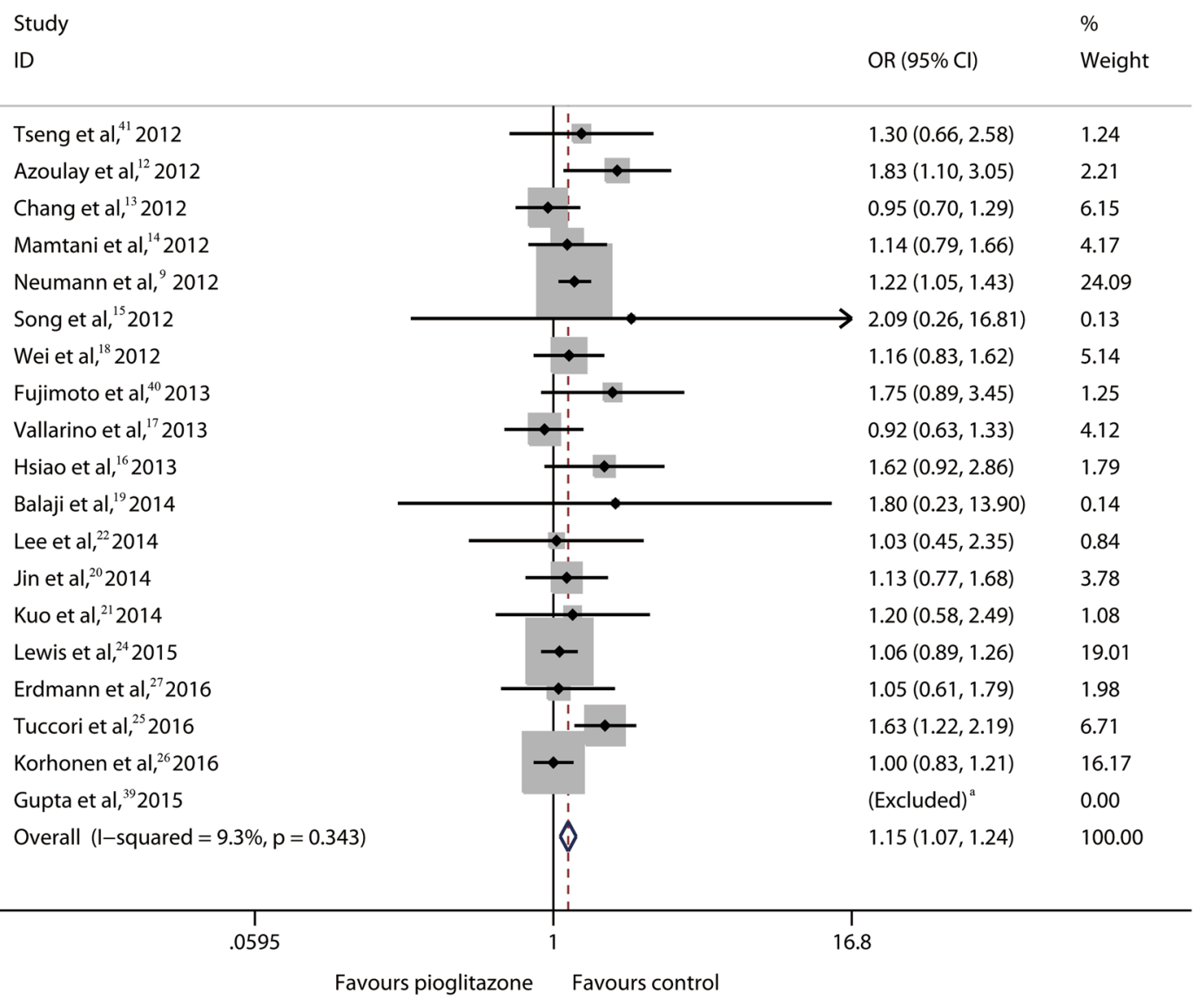

Figure 2. Bladder Cancer Risks Related to Pioglitazone Use Versus Control for Patients with DM in Global from OB Studies Abbreviations: DM, diabetes mellitus. ${ }^{a}$ Given that both the exposed and control groups did not report bladder cancer incidence, the OR was not estimable.

4 RCTs were identified; thus, at first the OB studies were analyzed. The results showed that the risk for bladder cancer risk increased by $15 \%$ (OR, 1.15; 95\% CI, 1.07-1.24; $P<0.001)$ (Fig. 2), indicating that pioglitazone use was associated with overall bladder cancer risk based on the existing evidence.

Study type and pioglitazone related bladder cancer risk at the global level. Next, to explore the reason behind the inconsistent outcomes observed in previous individual studies, the potential confounders for study outcome, which are generally derived from study design (study type, adjustment factors, intervention measures, comparator agents, follow-up duration, and study population) ${ }^{30}$, were analyzed. In terms of the study type, cohort studies showed a positive result with regard to the relationship between pioglitazone and bladder cancer risk (OR, 1.14; 95\% CI, 1.05-1.24; $P=0.001$ ), whereas case control studies showed negative results (OR, 1.21; 95\% CI, 0.97-1.52; $P=0.10$ ) (Fig. 3), suggesting that study type might be a confounder for the global outcomes.

Adjustment factors and pioglitazone related bladder cancer risk at the global level. With regard to adjustment factors, the main cancer risk factors that are commonly adjusted in medication related cancer risk studies include age/sex, smoking, unhealthy diet and lack of physical activity, and the use of other medications ${ }^{49}$. Although the risk factor "unhealthy diet and lack of physical activity" could not be analyzed (refer to the limitations list in the Discussion section for the reasons), the remaining 3 factors were evaluated. The adjustment for both smoking and use of other medications did not influence the outcomes (smoking: adjusted, OR, 1.18; 95\% CI, 1.05-1.32; $P=0.006$ vs. non-adjusted, $\mathrm{OR}, 1.13 ; 95 \% \mathrm{CI}, 1.03-1.25 ; P=0.01$; use of other medications: adjusted, OR, 1.11; $95 \% \mathrm{CI}, 1.02-1.21 ; P=0.02$ vs. non-adjusted, $\mathrm{OR}, 1.33 ; 95 \% \mathrm{CI}, 1.12-1.57 ; P=0.001$ ), whereas adjustment for age/sex significantly affected the results (adjusted: OR, $1.15 ; 95 \% \mathrm{CI}, 1.06-1.24 ; P=0.001$ vs. non-adjusted: OR, $1.30 ; 95 \%$ CI, $0.86-1.96 ; P=0.22$ ) (Fig. 3). These results indicated that adjustment for age/sex might also be a confounder that affected previous outcomes, and the results of the studies adjusted for age/sex seem more credible.

Intervention measures and pioglitazone related bladder cancer risk at the global level. With regard to intervention measures, administration routes as well as the treatment duration and cumulative dose were commonly consid$\operatorname{ered}^{50-52}$. However, pioglitazone is only orally administrated, only the duration and cumulative dose were assessed in this study. Of the included OB trials, in 13, the duration or cumulative dose-response relation was evaluated, and in 8 , the unified categories were used. These 8 trials were analyzed. Increased risks of bladder cancer were identified in longer duration and larger cumulative dose subgroups (duration: 12-24 months, OR, 1.29; 95\% CI, 
Study

ID

$\mathrm{OR}(95 \% \mathrm{Cl})$

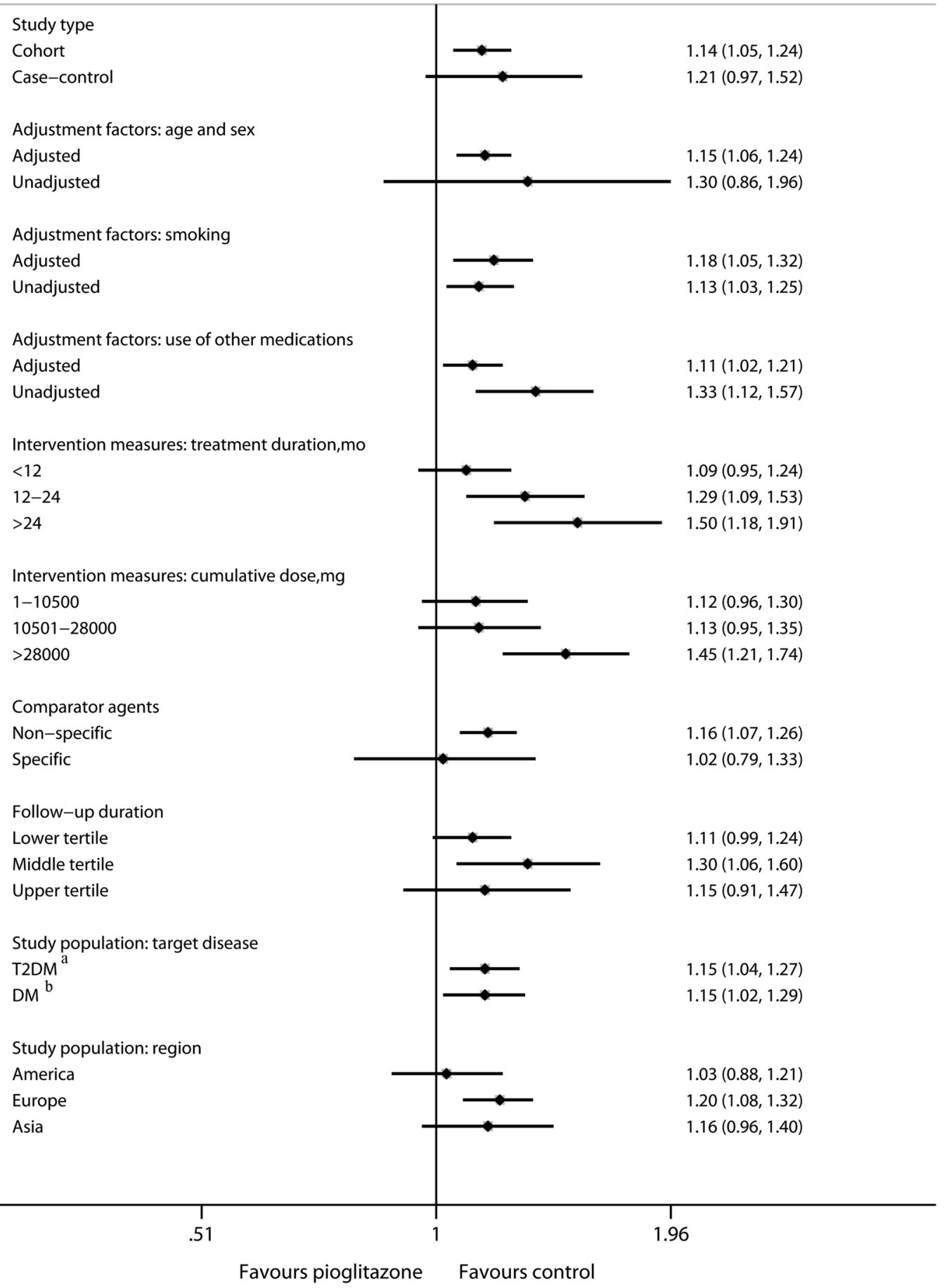

Figure 3. Subgroup Analyses of Bladder Cancer Risk Related to Pioglitazone Use Versus Control for Patients with DM in Global from OB Studie. Abbreviations: T2DM, type 2 diabetes mellitus; T1DM, type 1 diabetes mellitus. ${ }^{\text {aW }}$ ith T1DM excluded. ${ }^{\text {b} W i t h ~ T 1 D M ~ i n c l u d e d . ~}$

$1.09-1.53 ; P=0.003 ;>24$ months, OR, $1.50 ; 95 \% \mathrm{CI}, 1.18-1.91 ; P=0.001$; cumulative dose: $>28,000 \mathrm{mg}, \mathrm{OR}$, $1.45 ; 95 \%$ CI, 1.21-1.74; $P<0.001$ ), while the shorter duration and smaller cumulative dose subgroups did not increased risks of bladder cancer (duration: $<12$ months, OR, $1.09 ; 95 \% \mathrm{CI}, 0.95-1.24 ; P=0.21$; cumulative dose: $1-10,500 \mathrm{mg}$, OR, 1.12; 95\% CI, 0.96-1.30; $P=0.17 ; 10,501-28,000 \mathrm{mg}, \mathrm{OR}, 1.13 ; 95 \% \mathrm{CI}, 0.95-1.35 ; P=0.16$ ) (Fig. 3). These results indicated that the treatment duration and cumulative dose might also be confounders, and that long durations and large cumulative doses of pioglitazone use should be carefully monitored. 
Comparator agents and pioglitazone-related bladder cancer risk at the global level. The medication used in the control group can also be a potential confounder ${ }^{53}$. Of the included $\mathrm{OB}$ trials, non-specific agents were used in the control groups of 17 studies, and specific agents (insulin and rosiglitazone) were reported in 2. A positive relationship was identified in the non-specific comparator subgroup (OR, 1.16; 95\% CI, 1.07-1.26; $P<0.001$ ), whereas the result was negative in the specific comparator subgroup (OR, 1.02; 95\% CI, 0.79-1.33; $P=0.86$ ) (Fig. 3), indicating that comparator agents might influence the outcomes. Moreover, insulin and rosiglitazone have been reported to potentially influence bladder cancer risks ${ }^{54,55}$, this likely leads to the incorrect estimation of the association between pioglitazone use and bladder cancer risk. Thus, the results of studies that used non-specific agents as the control group might be more reasonable.

Follow-up duration and pioglitazone related bladder cancer risk at the global level. The follow-up duration mainly refers to the mean period of follow-up, as the maximum period cannot describe the maturity of the study data and the quality of follow-up ${ }^{56}$. This factor was analyzed using the tertiles method (the lower and upper cut-off points were 3.6 and 4.8 years, respectively) in our study. The middle tertile exhibited a positive association between pioglitazone and bladder cancer risk (OR, 1.30; 95\% CI, 1.06-1.60; $P=0.01)$, whereas the lower $(\mathrm{OR}, 1.11 ; 95 \%$ CI, 0.99-1.24; $P=0.08)$ and upper tertiles (OR, $1.15 ; 95 \% \mathrm{CI}, 0.91-1.47 ; P=0.25)$ did not show any association (Fig. 3), indicating that the follow-up duration might influence the outcomes. However, the pooled result of the upper tertile was inexplicable at the global level.

Study population and pioglitazone related bladder cancer risk at the global level. The study population commonly includes the target disease and the population region ${ }^{57}$. In terms of target disease, the relationship between pioglitazone use and bladder cancer risk was positive both in the T2DM (with T1DM excluded) and DM (with T1DM included) subgroups (T2DM: OR, 1.15; 95\% CI, 1.04-1.27; $P=0.006$ vs. DM: OR, 1.15; 95\% CI, 1.02-1.29; $P=0.02$ ) (Fig. 3). However, for the population region, the European region exhibited an increased risk of bladder cancer after pioglitazone exposure (OR, 1.20; 95\% CI, 1.08-1.32; $P<0.001$ ), whereas the results in both American (OR, 1.03; 95\% CI, 0.88-1.21; $P=0.68$ ) and Asian (OR, 1.16; 95\% CI, 0.96-1.40; $P=0.12$ ) regions were negative (Fig. 3), indicating that the population region might be a confounder at the global level.

Study design and pioglitazone related bladder cancer risk in different population regions. The population region is a confounder that influences outcomes objectively ${ }^{58-60}$. Therefore, we re-analyzed the potential confounders in different population regions. For American and Asian regions, the results were negative $(P>0.05)$, further confirming the negative pooled results (Fig. 4, Table 1). In the European region, the results were positive $(P<0.05)$ except for the subgroups unadjusted for age/sex (OR, 1.05; 95\% CI, 0.61-1.80; $P=0.86)$, moking $(\mathrm{OR}, 1.12 ; 95 \%$ $\mathrm{CI}, 1.00-1.26 ; P=0.052)$, the shortest treatment duration $(\mathrm{OR}, 1.08 ; 95 \% \mathrm{CI}, 0.87-1.34 ; P=0.49)$ and follow-up period (OR, 1.11; 95\% CI, 0.92-1.35; $P=0.28$ ), and specific agent (rosiglitazone) used in the control group (OR, $1.14 ; 95 \% \mathrm{CI}, 0.79-1.65 ; P=0.49)$. As mentioned above, the results of studies adjusted for age/sex and smoking, with longer treatment durations, and non-specific agents used in the control group were considered more credible; therefore, the positive results achieved in these subgroups in both Europe and globally further confirmed the positive pooled European and global results (Fig. 4, Table 1). Interestingly, the subgroup results of the longest follow-up were positive in the European populations (OR, 1.47; 95\% CI, 1.14-1.91; $P=0.003$ ) but negative in the American and Asian populations (OR, 1.03 ; 95\% CI, $0.89-1.20 ; P=0.68$ ). This finding might explain the inexplicable negative result of the longest follow-up subgroup at global level (Fig. 4, Table 1). Moreover, when the bladder cancer risk related to pioglitazone was considered based on different population regions, the study type no longer influenced the outcomes (Fig. 4, Table 1). Taken together, these results indicated that a positive relationship between pioglitazone and bladder cancer risk may be present in the European population. Thus, it is important to pay more attention to European patients, especially those in whom the treatment duration is long; the long-term effects of pioglitazone need to be monitored closely in these cases. In addition, future related studies should adjust for age/sex and smoking, and adopt non-specific agents in the control group.

Pioglitazone and other cancer risks. In addition to bladder cancer, pioglitazone exposure has also been reported to be associated with prostate and pancreatic cancer risks ${ }^{24,27}$. To examine the relationship between pioglitazone and the other cancers types, 16 site-specific cancers assessed in 12 related OB studies were analyzed. However, one-third of the results of the initial analysis yielded significant heterogeneities $\left(I^{2}>50 \%\right.$, esophagus, thyroid, brain and biliary cancers were only reported by one study each, and it was not possible to test heterogeneity, eFigure 1 in the Supplement). Therefore, sensitivity analyses were conducted using the "leave one out" approach ${ }^{38}$, and one study (Vallarino et al. ${ }^{17}$ ) was identified as a common contributor for these heterogeneities $\left(I^{2}\right.$ decreased to $\leq 32 \%$ with Vallarino et al. ${ }^{17}$ excluded) (Fig. 5). The re-summary results showed that pioglitazone use was associated with increased risks of prostate and pancreatic cancer (prostate: $\mathrm{OR}, 1.12 ; 95 \% \mathrm{CI}, 1.02-1.23 ; P=0.02$; pancreatic: OR, $1.33 ; 95 \% \mathrm{CI}, 1.12-1.57 ; P=0.001$ ), and decreased risks of liver and brain cancer (liver: OR, 0.83 ; 95\% CI, 0.73-0.96; $P=0.01$; brain: OR, 0.28; 95\% CI, 0.08-0.98; $P=0.047$, with only one report) (Fig. 5). These results indicated that pioglitazone use might also be related to other cancer risks.

Part 2: Reanalysis using OB studies and RCTs. Heretofore, only 4 RCTs related to pioglitazone and bladder cancer risk and a positive pooled result of RCTs have been reported ${ }^{61}$. Therefore, we combined all the OB studies with the 4 RCTs and reanalyzed the results. Given that the 4 RCTs were not involved in the adjustment factors and intervention measures analyses, we included comparator agents, follow-up duration, and study population in the subgroup analyses. As for other cancers, 17 site-specific cancers were analyzed. All the re-analytical results with OB studies plus RCTs were similar to those obtained from the OB studies (eFigure 2-5 in the Supplement). 
Study

ID

$\mathrm{OR}(95 \% \mathrm{Cl})$

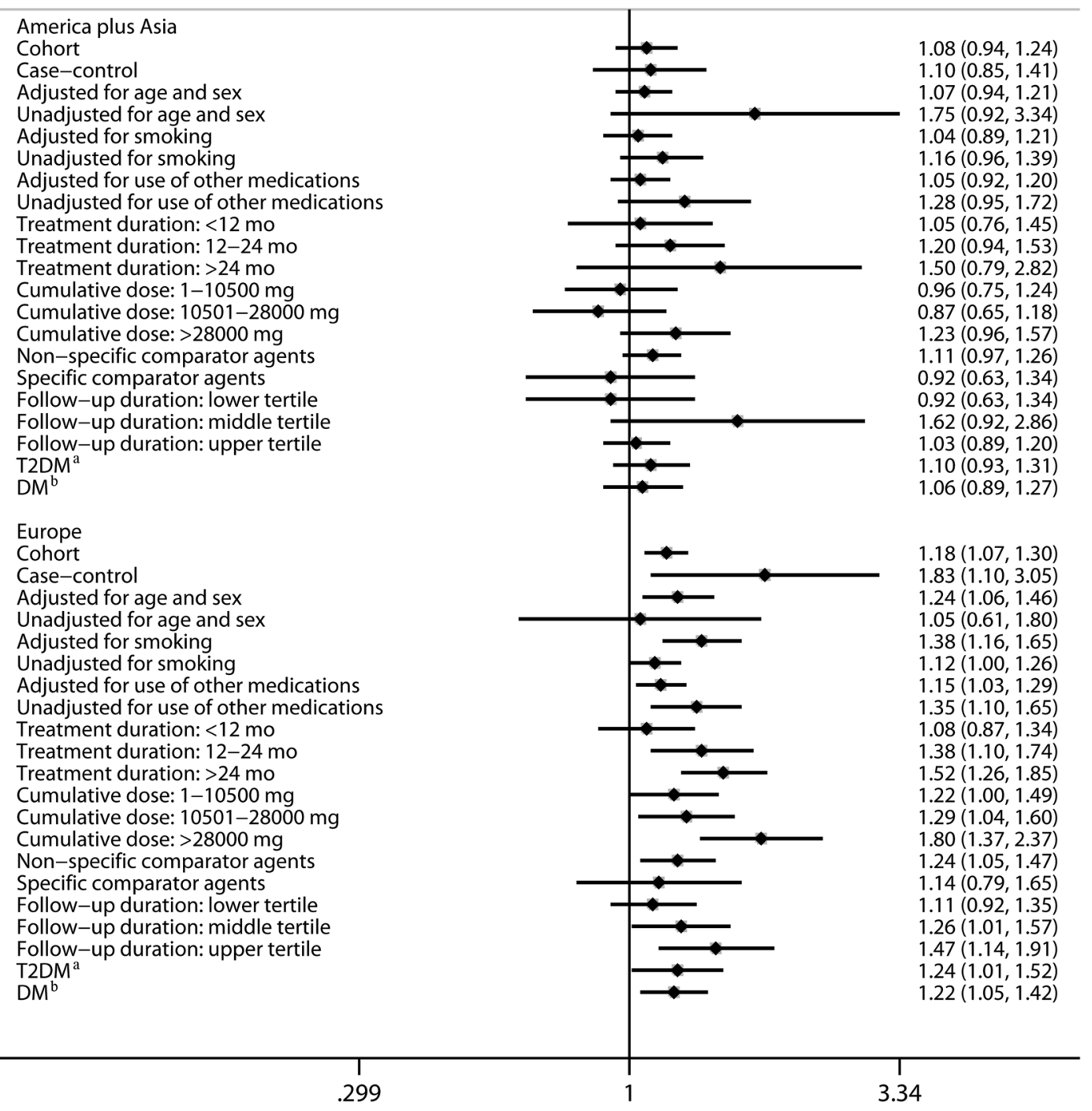

Favours pioglitazone Favours control

Figure 4. Subgroup Analyses of Bladder Cancer Risk Related to Pioglitazone Use Versus Control for Patients with DM in Europe, and America plus Asia, Respectively from OB Studies. ${ }^{\text {aW }}$ ith T1DM excluded. ${ }^{\text {b With }}$ T1DM included.

Sensitivity Analysis. For overall bladder cancer risk, sensitivity analyses were performed both using "study quality" analysis (eTable 2 in the supplement) and using the "leave one out" approach, and none of the results showed significant changes. For the subgroup analyses of bladder cancer risk, "study quality" sensitivity analysis was conducted, and the results remained similar. With regard to risks for other cancer types, a sensitivity analysis was conducted using the "leave one out" approach, the results of which are presented in the "Pioglitazone and other cancer risks" section of the Results.

Publication Bias Analysis. No significant bias was detected by Egger's test $(P=0.22)$ or Begg's test $(P=0.57$ [continuity corrected]) (eFigure 6, 7 in the supplement).

\section{Discussion}

Global safety concerns about the association of pioglitazone use with bladder cancer risk have been present and debated since the PROspective pioglitAzone Clinical Trial in macroVascular Events (PROactive) study was conducted, more than a decade ago $^{10}$. Recently, the discordant results obtained from several large-population studies further increased the uncertainty ${ }^{24-26}$. Here, we identified certain regional differences that might be extremely important factors that contribute to the inconsistent outcomes of the previous studies.

Our analysis suggested that pioglitazone use was associated with bladder cancer risk at the global level (15\% increase), which was consistent with the results of most of the previous meta-analyses ( $17 \%$ to $23 \%$ increase) $0^{51,52,61-64}$. 


\begin{tabular}{|c|c|c|c|c|c|c|c|c|c|}
\hline & \multicolumn{3}{|c|}{ Global } & \multicolumn{6}{|c|}{ Sub-regional } \\
\hline & \multirow[b]{2}{*}{ Outcome $^{a}$} & \multirow[b]{2}{*}{ OR $(95 \% \mathrm{CI})$} & \multirow{2}{*}{$P$-Value } & \multicolumn{3}{|c|}{ American plus Asian Regions } & \multicolumn{3}{|c|}{ European Region } \\
\hline & & & & Outcome $^{\mathrm{a}}$ & OR $(95 \% \mathrm{CI})$ & $P$-Value & Outcome $^{a}$ & OR $(95 \% \mathrm{CI})$ & $P$-Value \\
\hline \multicolumn{10}{|l|}{ Study type } \\
\hline Cohort & + & $1.14(1.05,1.24)$ & 0.001 & - & $1.08(0.94,1.24)$ & 0.26 & + & $1.18(1.07,1.30)$ & 0.001 \\
\hline Case-control & - & $1.21(0.97,1.52)$ & 0.10 & - & $1.10(0.85,1.41)$ & 0.47 & + & $1.83(1.10,3.05)$ & 0.02 \\
\hline \multicolumn{10}{|c|}{ Adjustment factors } \\
\hline \multicolumn{10}{|l|}{ Age and sex } \\
\hline Adjusted & + & $1.15(1.06,1.24)$ & 0.001 & - & $1.07(0.94,1.21)$ & 0.30 & + & $1.24(1.06,1.46)$ & 0.009 \\
\hline Unadjusted & - & $1.30(0.86,1.96)$ & 0.22 & - & $1.75(0.92,3.34)$ & 0.09 & - & $1.05(0.61,1.80)$ & 0.86 \\
\hline \multicolumn{10}{|l|}{ Smoking } \\
\hline Adjusted & + & $1.18(1.05,1.32)$ & 0.006 & - & $1.04(0.89,1.21)$ & 0.64 & + & $1.38(1.16,1.65)$ & $<0.001$ \\
\hline Unadjusted & + & $1.13(1.03,1.25)$ & 0.01 & - & $1.16(0.96,1.39)$ & 0.13 & - & $1.12(1.00,1.26)$ & 0.052 \\
\hline \multicolumn{10}{|c|}{ Use of other medications } \\
\hline Adjusted & + & $1.11(1.02,1.21)$ & 0.02 & - & $1.05(0.92,1.20)$ & 0.45 & + & $1.15(1.03,1.29)$ & 0.01 \\
\hline Unadjusted & + & $1.33(1.12,1.57)$ & 0.001 & - & $1.28(0.95,1.72)$ & 0.11 & + & $1.35(1.10,1.65)$ & 0.004 \\
\hline \multicolumn{10}{|c|}{ Intervention measures } \\
\hline \multicolumn{10}{|c|}{ Treatment duration, mo } \\
\hline$<12$ & - & $1.09(0.95,1.24)$ & 0.21 & - & $1.05(0.76,1.45)$ & 0.78 & - & $1.08(0.87,1.34)$ & 0.49 \\
\hline $12-24$ & + & $1.29(1.09,1.53)$ & 0.003 & - & $1.20(0.94,1.53)$ & 0.14 & + & $1.38(1.10,1.74)$ & 0.006 \\
\hline$>24$ & + & $1.50(1.18,1.91)$ & 0.001 & - & $1.50(0.79,2.82)$ & 0.21 & + & $1.52(1.26,1.85)$ & $<0.001$ \\
\hline \multicolumn{10}{|c|}{ Cumulative dose, mg } \\
\hline $1-10500$ & - & $1.12(0.96,1.30)$ & 0.17 & - & $0.96(0.75,1.24)$ & 0.78 & + & $1.22(1.00,1.49)$ & 0.048 \\
\hline $10501-28000$ & - & $1.13(0.95,1.35)$ & 0.16 & - & $0.87(0.65,1.18)$ & 0.37 & + & $1.29(1.04,1.60)$ & 0.02 \\
\hline$>28000$ & + & $1.45(1.21,1.74)$ & $<0.001$ & - & $1.23(0.96,1.57)$ & 0.10 & + & $1.80(1.37,2.37)$ & $<0.001$ \\
\hline \multicolumn{10}{|c|}{ Comparator agents } \\
\hline \multicolumn{10}{|c|}{ Agents use in control group } \\
\hline Specific & + & $1.16(1.07,1.26)$ & $<0.001$ & - & $1.11(0.97,1.26)$ & 0.12 & + & $1.24(1.05,1.47)$ & 0.01 \\
\hline Non-specific & - & $1.02(0.79,1.33)$ & 0.86 & - & $0.92(0.63,1.34)$ & 0.66 & - & $1.14(0.79,1.65)$ & 0.49 \\
\hline \multicolumn{10}{|c|}{ Follow-up Duration } \\
\hline \multicolumn{10}{|c|}{ Mean period of follow-up, $y$} \\
\hline Lower tertile, $<3.6$ & - & $1.11(0.99,1.24)$ & 0.08 & - & $0.92(0.63,1.34)$ & 0.66 & - & $1.11(0.92,1.35)$ & 0.28 \\
\hline $\begin{array}{l}\text { Middle tertile, } \\
3.6-4.8\end{array}$ & + & $1.30(1.06,1.60)$ & 0.013 & - & $1.62(0.92,2.86)$ & 0.10 & + & $1.26(1.01,1.57)$ & 0.04 \\
\hline Upper tertile, $\geq 4.8$ & - & $1.15(0.91,1.47)$ & 0.25 & - & $1.03(0.89,1.20)$ & 0.68 & + & $1.47(1.14,1.91)$ & 0.003 \\
\hline \multicolumn{10}{|c|}{ Study population } \\
\hline \multicolumn{10}{|l|}{ Target disease } \\
\hline $\mathrm{T}_{2} \mathrm{DM}^{\mathrm{b}}$ & + & $1.15(1.04,1.27)$ & 0.006 & - & $1.10(0.93,1.31)$ & 0.24 & + & $1.24(1.05,1.47)$ & 0.01 \\
\hline $\mathrm{DM}^{\mathrm{c}}$ & + & $1.15(1.02,1.29)$ & 0.02 & - & $1.06(0.89,1.27)$ & 0.48 & + & $1.14(0.79,1.65)$ & 0.49 \\
\hline \multicolumn{10}{|c|}{ Study population region } \\
\hline America & - & $1.03(0.88,1.21)$ & 0.68 & NA & NA & NA & NA & NA & NA \\
\hline Europe & +1 & $1.20(1.08,1.32)$ & $<0.001$ & NA & NA & NA & NA & NA & NA \\
\hline Asia & - & $1.16(0.96,1.40)$ & 0.12 & NA & NA & NA & NA & NA & NA \\
\hline
\end{tabular}

Table 1. Bladder Cancer Risk Related to Pioglitazone Use in Patients with DM Compared Globally and Regionally from OB Studies. Abbreviations: DM, diabetes mellitus; OB, observational studies; T2DM, type 2 diabetes; T1DM, type 1 diabetes; NA, not available. ${ }^{a}$ The outcome includes statistical increased risk of bladder cancer $(+)$ and non-association (-) related to pioglitazone use. ${ }^{b}$ With T1DM excluded. ${ }^{c}$ With T1DM included.

In addition, the negative results were achieved from the subgroups unadjusted for age/sex, short treatment durations and small cumulative doses, and the specific agents (insulin and rosiglitazone) used as comparators during the stratified analysis with potential confounders. These also strengthen the positive relationship, as adjusted results are more credible, and diabetic patients require long-term treatment ${ }^{65}$, and hence insulin and rosiglitazone may influence bladder cancer risk ${ }^{54,55}$.

Most importantly, our results indicated for the first time that the association of pioglitazone use and bladder cancer risk exhibits a significant regional variation. While the pooled and stratified results were negative in the American and Asian regions, results from studies conducted in the European region showed a striking positivity from the pooled and most of the stratified analyses. Although the subgroups unadjusted for age/sex and smoking, short treatment duration and follow-up period, and rosiglitazone used in the control group showed negative results, based on the reasons mentioned above, the results further confirmed a positive relationship in European populations. This regional difference might explain the discordant results of several previous studies, such as the KPNC and 


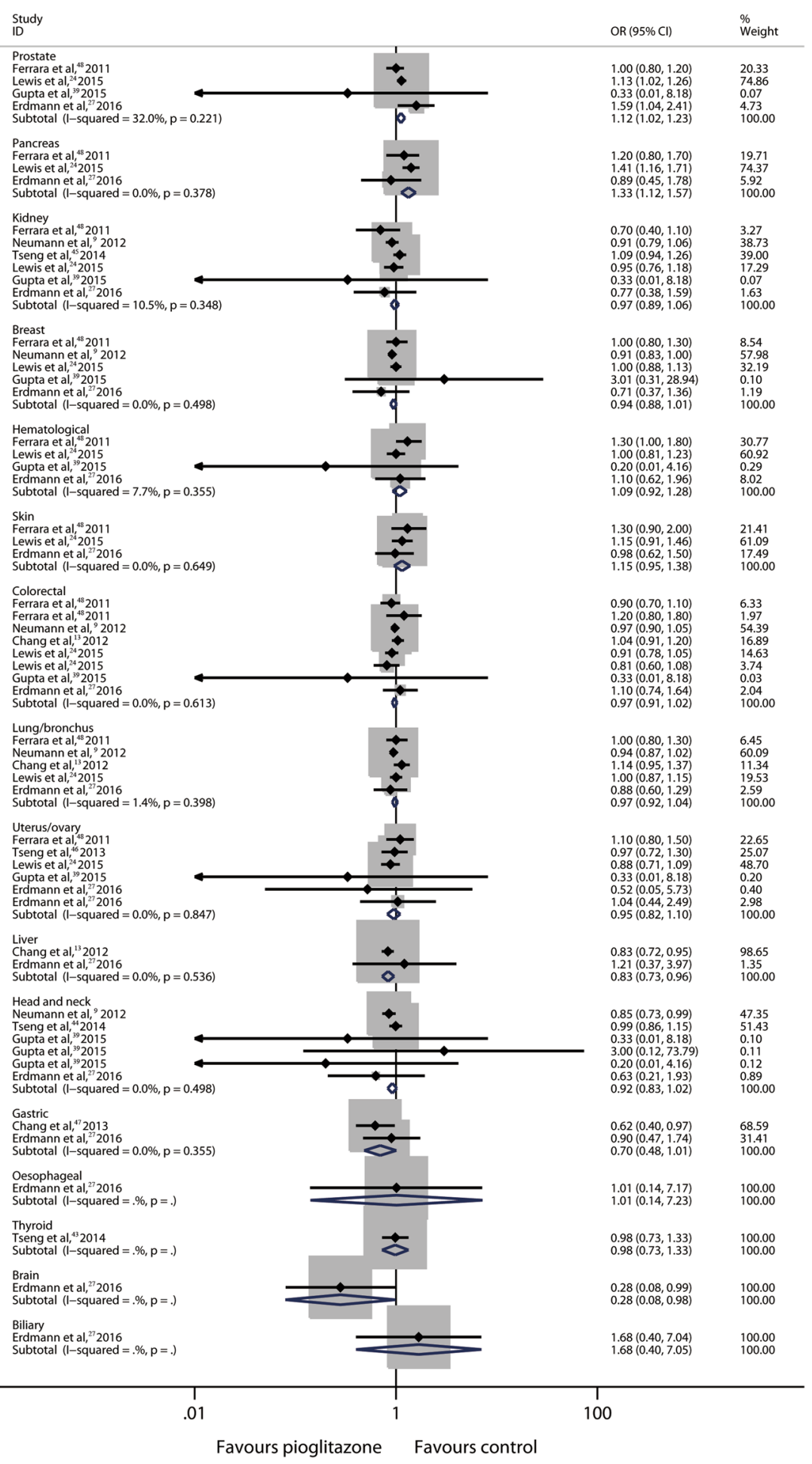

Figure 5. Other Cancer Risks in Patients with DM Receiving Pioglitazone Versus Control from OB Studies ${ }^{\mathrm{a}}$. ${ }^{a}$ With Vallarino et al. ${ }^{17}$ excluded.

$\mathrm{UKCPRD}^{24,25}$. Similarly, the risk factors for stroke, disabling sequelae from bacterial meningitis, and obesity were also reported to have regional differences ${ }^{58-60}$. Interestingly, when the relationship was considered in terms of different population regions, the study type no longer influenced the outcomes, and the inexplicable results of follow-up duration analysis at the global level could be interpreted reasonably. Based on these findings, we suggest that the bladder cancer risk related to pioglitazone should be considered according to the different population regions, and the European population require more attention. Notably, although we identified the regional differences across continents, the possibility of variation among different countries and regions may exist. Whether the regional 
differences that we observed in this study are associated with race and ethnicity remain unclear, as only the KPNC study ${ }^{24}$ described the racial composition of its study population. Further studies are required to clarify these issues.

Moreover, when performing future studies, the authors need to be aware of the long-term effects of prolonged pioglitazone use and should adjust for age/sex and smoking and adopt non-specific agents in the control group. Although our results indicated that the study design did not influence the outcomes of the American and Asian populations, it still had an effect on the European population. In the European population, positive results were achieved with long treatment durations ( $>12$ months) and follow-up periods ( $\geq 3.6$ years); therefore, we should pay more attention to this population. In addition, adjustment for age/sex and smoking, and the specific comparator agent (rosiglitazone) influenced the outcomes; therefore, these factors should also be noticed in future related studies.

Our results indicated that pioglitazone use might also be related to increased risk for other cancer types, such as prostate and pancreatic cancer, which is consistent with the observations of the KPNC study ${ }^{24}$, and decreased risks for liver and brain cancer. Additionally, one study recently suggested that pioglitazone might influence cancer progression ${ }^{66}$. However, studies on these other cancers are very limited and more studies are needed to answer several key questions in this field: Does pioglitazone influence the risk and/or progression of other cancers? Does the association between pioglitazone use and risk for other cancer types show regional differences? If so, what is the mechanism behind the role of pioglitazone in tumorigenesis?

Our study has important implications in future clinical practice and trials in that (1) more attention regarding bladder cancer risk related to pioglitazone should be given to European, especially those with long treatment durations and a large cumulative dose; (2) studies should adjust for age/sex and smoking, and adopt non-specific agents in the control group; and (3) the risks of other site-specific cancers, such as prostate, pancreatic, liver and brain cancer, need to be addressed.

These are several limitations to our study. First, among the main cancer risk factors, the factor "unhealthy diet and lack of physical activity" was not analyzed because it was reported by only one study ${ }^{12}$ in the form of alcohol intake. Second, some factors that were adjusted for in previous individual trials were not analyzed for the following reasons. Only one ${ }^{18}$ and two trials ${ }^{22,24}$ have adjusted for body mass index (BMI) and income, respectively; therefore, the data are inadequate for meaningful analyses. The comorbidities selected for adjustment different from trial to trial, and the diabetes duration and date of cohort entry were defined inconsistently among the trials ${ }^{9,12-22,24-27,39-41}$; thus, these 3 factors were also unsuitable for analysis. Only 3 trials adjusted for hemoglobin Alc concentration $(\mathrm{HbAlc})^{12,24,25}$. Although the analyzed results were accordant with our conclusions, more data might be required for further analysis. The results obtained from analysis at the global level showed that adjustment for HbAlc affected the outcome (adjusted: OR, 1.40; 95\% CI, 0.98-2.02; $P=0.07$ vs. non-adjusted: OR, 1.12; $95 \%$ CI, 1.03-1.23; $P=0.01$, efigure 8 in the supplement). However, only 3 adjusted trials were included, of which $2^{12,25}$ were conducted in Europe and $1^{24}$ was conducted in America; thus, this regional difference might contribute to the negative pooled results of the adjusted trials at the global level. When the association was analyzed by region, the results of both the $\mathrm{HbAlc}$-adjusted and unadjusted groups were positive in the European regions (adjusted: OR, 1.68; 95\% CI, 1.30-2.16; $P<0.001$ vs. non-adjusted: OR, 1.13; 95\% CI, $1.01-1.25 ; P=0.03$ ) but negative in the American and Asian regions (adjusted: OR, 1.06; 95\% CI, 0.89-1.26; $P=0.51$ vs. non-adjusted: OR, $1.11 ; 95 \% \mathrm{CI}, 0.94-1.31 ; P=0.23$ ) (efigure 9 in the supplement), which further confirmed our conclusions. Third, the variation of diagnostic criteria for bladder cancer and other cancers among these studies could not be assessed in our study. Finally, most of the included studies were observational in design and patient-level data for each of the studies could not be obtained due to authorization limit. However, most of these studies have adjusted for major potential confounders, such as age/sex, smoking, and comorbidities, for bias control.

\section{Conclusions}

In summary, the risk of bladder cancer with pioglitazone use might needs to be considered in the European population, and patients with a longer treatment duration ( $>12$ months) or a larger cumulative dose $(>28,000 \mathrm{mg})$ might should be followed up more carefully. The long-term effects ( $\geq 3.6$ years) of pioglitazone might also need to be noted. In addition, future related studies should adjust for age/sex and smoking and adopt non-specific agents in the control group. Moreover, pioglitazone use may relate to risks for other cancer types.

Access to research materials. Information about how the data can be accessed is available from the corresponding author.

\section{References}

1. Vamecq, J. \& Latruffe, N. Medical significance of peroxisome proliferator-activated receptors. Lancet 354, 141-148, https://doi. org/10.1016/S0140-6736(98)10364-1 (1999).

2. Campbell, I. W. The Clinical Significance of PPAR Gamma Agonism. Curr Mol Med 5, 349-363 (2005).

3. Bjornsson, E. \& Olsson, R. Suspected drug-induced liver fatalities reported to the WHO database. Dig Liver Dis 38, 33-38, https:// doi.org/10.1016/j.dld.2005.06.004 (2006).

4. Mitka, M. Panel recommends easing restrictions on rosiglitazone despite concerns about cardiovascular safety. JAMA 310, 246-247, https://doi.org/10.1001/jama.2013.8141 (2013).

5. Woodcock, J. \& Sharfstein, J. M. \& Hamburg, M. Regulatory action on rosiglitazone by the U.S. Food and Drug Administration. N Engl J Med 363, 1489-1491, https://doi.org/10.1056/NEJMp1010788 (2010).

6. Cusi, K. et al. Long-Term Pioglitazone Treatment for Patients With Nonalcoholic Steatohepatitis and Prediabetes or Type 2 Diabetes Mellitus: A Randomized Trial. Ann Intern Med 165, 305-315, https://doi.org/10.7326/M15-1774 (2016).

7. Kernan, W. N. et al. Pioglitazone after Ischemic Stroke or Transient Ischemic Attack. N Engl J Med 374, 1321-1331, https://doi. org/10.1056/NEJMoa1506930 (2016).

8. Lewis, J. D. et al. Risk of bladder cancer among diabetic patients treated with pioglitazone: interim report of a longitudinal cohort study. Diabetes Care 34, 916-922, https://doi.org/10.2337/dc10-1068 (2011). 
9. Neumann, A. et al. Pioglitazone and risk of bladder cancer among diabetic patients in France: a population-based cohort study. Diabetologia 55, 1953-1962, https://doi.org/10.1007/s00125-012-2538-9 (2012).

10. Dormandy, J. A. et al. Secondary prevention of macrovascular events in patients with type 2 diabetes in the PROactive Study (PROspective pioglitAzone Clinical Trial In macroVascular Events): a randomised controlled trial. Lancet 366, 1279-1289, https:// doi.org/10.1016/S0140-6736(05)67528-9 (2005).

11. Tseng, C. H. Diabetes and risk of bladder cancer: a study using the National Health Insurance database in Taiwan. Diabetologia 54, 2009-2015, https://doi.org/10.1007/s00125-011-2171-z (2011).

12. Azoulay, L. et al. The use of pioglitazone and the risk of bladder cancer in people with type 2 diabetes: nested case-control study. $B M J$ 344, e3645, https://doi.org/10.1136/bmj.e3645 (2012).

13. Chang, C. H. et al. Association of thiazolidinediones with liver cancer and colorectal cancer in type 2 diabetes mellitus. Hepatology 55, 1462-1472, https://doi.org/10.1002/hep.25509 (2012).

14. Mamtani, R. et al. Association between longer therapy with thiazolidinediones and risk of bladder cancer: a cohort study. J Natl Cancer Inst 104, 1411-1421, https://doi.org/10.1093/jnci/djs328 (2012).

15. Song, S. O. et al. The risk of bladder cancer in korean diabetic subjects treated with pioglitazone. Diabetes Metab J 36, 371-378, https://doi.org/10.4093/dmj.2012.36.5.371 (2012).

16. Hsiao, F. Y., Hsieh, P. H., Huang, W. F., Tsai, Y. W. \& Gau, C. S. Risk of bladder cancer in diabetic patients treated with rosiglitazone or pioglitazone: a nested case-control study. Drug Saf 36, 643-649, https://doi.org/10.1007/s40264-013-0080-4 (2013).

17. Vallarino, C. et al. Comparing pioglitazone to insulin with respect to cancer, cardiovascular and bone fracture endpoints, using propensity score weights. Clin Drug Investig 33, 621-631, https://doi.org/10.1007/s40261-013-0106-9 (2013).

18. Wei, L., MacDonald, T. M. \& Mackenzie, I. S. Pioglitazone and bladder cancer: a propensity score matched cohort study. Br J Clin Pharmacol 75, 254-259, https://doi.org/10.1111/j.1365-2125.2012.04325.x (2012).

19. Balaji, V., Seshiah, V., Ashtalakshmi, G., Ramanan, S. G. \& Janarthinakani, M. A retrospective study on finding correlation of pioglitazone and incidences of bladder cancer in the Indian population. Indian J Endocrinol Metab 18, 425-427, https://doi. org/10.4103/2230-8210.131223 (2014)

20. Jin SM, S. S. \& Jung, C. H. Risk of bladder cancer among patients with diabetes treated with a $15 \mathrm{mg}$ pioglitazone dose in Korea: a multi-center retrospective cohort study. J Korean Med Sci 29, 5 (2014).

21. Kuo H W, T. M., Ho, S. C. \& Yang, C. Y. Pioglitazone use and the risk of bladder cancer. Kaohsiung J Med Sci 30, 4 (2014).

22. Lee, M. Y., Hsiao, P. J., Yang, Y. H., Lin, K. D. \& Shin, S. J. The association of pioglitazone and urinary tract disease in type 2 diabetic Taiwanese: bladder cancer and chronic kidney disease. PLoS One 9, e85479, https://doi.org/10.1371/journal.pone.0085479 (2014).

23. Levin, D. et al. Pioglitazone and bladder cancer risk: a multipopulation pooled, cumulative exposure analysis. Diabetologia 58, 493-504, https://doi.org/10.1007/s00125-014-3456-9 (2015)

24. Lewis, J. D. et al. Pioglitazone Use and Risk of Bladder Cancer and Other Common Cancers in Persons With Diabetes. JAMA 314, 265-277, https://doi.org/10.1001/jama.2015.7996 (2015).

25. Tuccori, M. et al. Pioglitazone use and risk of bladder cancer: population based cohort study. BMJ 352, i1541, https://doi. org/10.1136/bmj.i1541 (2016).

26. Korhonen, P. et al. Pioglitazone use and risk of bladder cancer in patients with type 2 diabetes: retrospective cohort study using datasets from four European countries. Bmj 354 (2016).

27. Erdmann, E., Harding, S., Lam, H. \& Perez, A. Ten-year observational follow-up of PROactive: a randomized cardiovascular outcomes trial evaluating pioglitazone in type 2 diabetes. Diabetes Obes Metab 18, 266-273, https://doi.org/10.1111/dom.12608 (2016).

28. Wells, G.A. et al. The Newcastle-Ottawa scale (NOS) for assessing the quality of nonrandomised studies in meta-analyses, http://www. ohri.ca/programs/clinical_epidemiology/oxford.asp.

29. Higgins, J. P. et al. Cochrane Bias Methods Group; Cochrane Statistical Methods Group. Cochrane Bias Methods Group; Cochrane Statistical Methods Group. The Cochrane Collaboration's tool for assessing risk of bias in randomised trials. Report No. 343, d5928 (2011).

30. Li, L. et al. Dipeptidyl peptidase-4 inhibitors and risk of heart failure in type 2 diabetes: systematic review and meta-analysis of randomised and observational studies. BMJ 352, i610, https://doi.org/10.1136/bmj.i610 (2016).

31. Greenland, S. Quantitative methods in the review of epidemiologic literature. Epidemiol Rev 9, 1-30 (1987).

32. DerSimonian, R. \& Laird, N. Meta-analysis in clinical trials. Control Clin Trials 7, 177-188 (1986).

33. Stroup, D. F. et al. Meta-analysis of observational studies in epidemiology: a proposal for reporting. Meta-analysis Of Observational Studies in Epidemiology (MOOSE) group. JAMA 283, 2008-2012 (2000).

34. Moher, D., Liberati, A., Tetzlaff, J., Altman, D. G. \& Group, P. Preferred reporting items for systematic reviews and meta-analyses: the PRISMA statement. BMJ 339, b2535, https://doi.org/10.1136/bmj.b2535 (2009).

35. Egger, M., Davey Smith, G., Schneider, M. \& Minder, C. Bias in meta-analysis detected by a simple, graphical test. BMJ 315, 629-634 (1997).

36. Begg, C. B. \& Mazumdar, M. Operating characteristics of a rank correlation test for publication bias. Biometrics 50, 1088-1101 (1994).

37. Higgins, J. P., Thompson, S. G., Deeks, J. J. \& Altman, D. G. Measuring inconsistency in meta-analyses. BMJ 327, 557-560, https:// doi.org/10.1136/bmj.327.7414.557 (2003).

38. Greenhouse JB, L. S. The handbook of research synthesis and meta-analysis. 2nd edn, 423 (Russell Sage Foundation, 2009).

39. Gupta, S. et al. Pioglitazone and the risk of bladder cancer: An Indian retrospective cohort study. Indian J Endocrinol Metab 19, 639-643, https://doi.org/10.4103/2230-8210.163187 (2015).

40. Fujimoto, K. et al. Possible link of pioglitazone with bladder cancer in Japanese patients with type 2 diabetes. Diabetes Res Clin Pract 99, e21-23, https://doi.org/10.1016/j.diabres.2012.11.013 (2013).

41. Tseng, C. H. Pioglitazone and bladder cancer: a population-based study of Taiwanese. Diabetes Care 35, 278-280, https://doi. org/10.2337/dc11-1449 (2012).

42. Nissen, S. E. et al. Comparison of pioglitazone vs glimepiride on progression of coronary atherosclerosis in patients with type 2 diabetes: the PERISCOPE randomized controlled trial. JAMA 299, 1561-1573, https://doi.org/10.1001/jama.299.13.1561 (2008).

43. Tseng, C. H. Pioglitazone and thyroid cancer risk in Taiwanese patients with type 2 diabetes 2 . J Diabetes 6, 448-450, https://doi. org/10.1111/1753-0407.12149 (2014).

44. Tseng, C. H. Pioglitazone and oral cancer risk in patients with type 2 diabetes. Oral Oncol 50, 98-103, https://doi.org/10.1016/j. oraloncology.2013.10.015 (2014).

45. Tseng, C. H. Pioglitazone does not affect the risk of kidney cancer in patients with type 2 diabetes. Metabolism 63, 1049-1055, https://doi.org/10.1016/j.metabol.2014.04.014 (2014).

46. Tseng, C. H. Pioglitazone does not affect the risk of ovarian cancer: analysis of a nationwide reimbursement database in Taiwan. Gynecol Oncol 131, 135-139, https://doi.org/10.1016/j.ygyno.2013.07.087 (2013).

47. Chang, S. S. \& Hu, H. Y. Association of thiazolidinediones with gastric cancer in type 2 diabetes mellitus: a population-based casecontrol study. BMC Cancer 13, 420, https://doi.org/10.1186/1471-2407-13-420 (2013).

48. Ferrara, A. et al. Cohort study of pioglitazone and cancer incidence in patients with diabetes. Diabetes Care 34, 923-929, https://doi. org/10.2337/dc10-1067 (2011).

49. World Health Organization. Cancer http://www.who.int/mediacentre/factsheets/fs297/en/ (2015). 
50. Seal, B. S., Anderson, S. \& Shermock, K. M. Factors Associated with Adherence Rates for Oral and Intravenous Anticancer Therapy in Commercially Insured Patients with Metastatic ColonCancer. J Manag Care Spec Pharm 22, 227-235, https://doi.org/10.18553/ jmcp.2016.22.3.227 (2016).

51. Zhu, Z., Shen, Z., Lu, Y., Zhong, S. \& Xu, C. Increased risk of bladder cancer with pioglitazone therapy in patients with diabetes: a meta-analysis. Diabetes Res Clin Pract 98, 159-163, https://doi.org/10.1016/j.diabres.2012.05.006 (2012).

52. Bosetti, C. et al. Cancer risk for patients using thiazolidinediones for type 2 diabetes: a meta-analysis. Oncologist 18, 148-156, https://doi.org/10.1634/theoncologist.2012-0302 (2013).

53. Nissen, S. E. \& Wolski, K. Effect of rosiglitazone on the risk of myocardial infarction and death from cardiovascular causes. $N$ Engl $J$ Med 356, 2457-2471, https://doi.org/10.1056/NEJMoa072761 (2007).

54. Newton, C. C., Gapstur, S. M., Campbell, P. T. \& Jacobs, E. J. Type 2 diabetes mellitus, insulin-use and risk of bladder cancer in a large cohort study. Int J Cancer 132, 2186-2191, https://doi.org/10.1002/ijc.27878 (2013).

55. Han, E. et al. Rosiglitazone Use and the Risk of Bladder Cancer in Patients With Type 2 Diabetes. Medicine (Baltimore) 95, e2786, https://doi.org/10.1097/MD.0000000000002786 (2016).

56. Shuster, J. J. Median follow-up in clinical trials. Journal of Clinical Oncology Official Journal of the American Society of Clinical Oncology 9, 191-192 (1991).

57. Rohrig, B., du Prel, J. B. \& Blettner, M. Study design in medical research: part 2 of a series on the evaluation of scientific publications. Dtsch Arztebl Int 106, 184-189, https://doi.org/10.3238/arztebl.2009.0184 (2009).

58. O'Donnell, M. J. et al. Global and regional effects of potentially modifiable risk factors associated with acute stroke in 32 countries (INTERSTROKE): a case-control study. Lancet 388, 761-775, https://doi.org/10.1016/S0140-6736(16)30506-2 (2016).

59. Edmond, K. et al. Global and regional risk of disabling sequelae from bacterial meningitis: a systematic review and meta-analysis. Lancet Infect Dis 10, 317-328, https://doi.org/10.1016/S1473-3099(10)70048-7 (2010).

60. Ng, M. et al. Global, regional, and national prevalence of overweight and obesity in children and adults during 1980-2013: a systematic analysis for the Global Burden of Disease Study 2013. Lancet 384, 766-781, https://doi.org/10.1016/S01406736(14)60460-8 (2014).

61. Turner, R. M. et al. Thiazolidinediones and associated risk of bladder cancer: a systematic review and meta-analysis. Br J Clin Pharmacol 78, 258-273, https://doi.org/10.1111/bcp.12306 (2014).

62. Ferwana, M. et al. Pioglitazone and risk of bladder cancer: a meta-analysis of controlled studies. Diabet Med 30, 1026-1032, https:// doi.org/10.1111/dme.12144(2013).

63. He, S. et al. Pioglitazone prescription increases risk of bladder cancer in patients with type 2 diabetes: an updated meta-analysis. Tumour Biol 35, 2095-2102, https://doi.org/10.1007/s13277-013-1278-x (2014).

64. Colmers, I. N., Bowker, S. L., Majumdar, S. R. \& Johnson, J. A. Use of thiazolidinediones and the risk of bladder cancer among people with type 2 diabetes: a meta-analysis. CMAJ 184, E675-683, https://doi.org/10.1503/cmaj.112102 (2012).

65. Walker, J. J., Johnson, J. A. \& Wild, S. H. Diabetes treatments and cancer risk: the importance of considering aspects of drug exposure. Lancet Diabetes Endocrinol 1, 132-139, https://doi.org/10.1016/S2213-8587(13)70028-7 (2013).

66. Darash-Yahana, M. et al. Breast cancer tumorigenicity is dependent on high expression levels of NAF-1 and the lability of its Fe-S clusters. Proc Natl Acad Sci USA 113, 10890-10895, https://doi.org/10.1073/pnas.1612736113 (2016).

\section{Acknowledgements}

Dr HT. Zheng had full access to all of the data in the study and takes responsibility for the integrity of the data and the accuracy of the data analysis. We thank $\mathrm{Na} \mathrm{Wu}$ (Department of Epidemiology, College of Preventive Medicine, Third Military Medical University, Chongqing, China), for additional statistical support, which was provided without compensation. This study was supported by grants from National Key R\&D Program of China (No. 2016YFC1101100), National Natural Science Foundation of China (No. 81471039, No. 81270893, No. 81228023, No. 81401601, and No. 81402202) and the Natural Science Foundation Project of Chongqing (CSTC2014jcyjjq10006, CSTC2012jjB10023, and CSTC2016jcyjA0518). Role of the Sponsors: The finding organizations had no role in the design and conduct of the study; the collection, management, analysis, and interpretation of the data; the preparation, review, or approval of the manuscript; or the decision to submit the manuscript for publication.

\section{Author Contributions}

Study concept and design: H.Q., Y.Z., Y.W., H.Z. Acquisition, analysis, or interpretation of data: H.Q., Y.Z., Y.W., H.Z. Drafting of the manuscript: H.Q., Y.Z. Critical revision of the manuscript for important intellectual content: X.R., Z.L., H.Z. Statistical analysis: Y.W., H.Q., R.Z. Obtained funding: Y.Z., H.Z. Administrative, technical, or material support: G.Y., H.Z. Study supervision: H.Z.

\section{Additional Information}

Supplementary information accompanies this paper at https://doi.org/10.1038/s41598-017-16074-1.

Competing Interests: The authors declare that they have no competing interests.

Publisher's note: Springer Nature remains neutral with regard to jurisdictional claims in published maps and institutional affiliations.

(c) (i) Open Access This article is licensed under a Creative Commons Attribution 4.0 International

License, which permits use, sharing, adaptation, distribution and reproduction in any medium or format, as long as you give appropriate credit to the original author(s) and the source, provide a link to the Creative Commons license, and indicate if changes were made. The images or other third party material in this article are included in the article's Creative Commons license, unless indicated otherwise in a credit line to the material. If material is not included in the article's Creative Commons license and your intended use is not permitted by statutory regulation or exceeds the permitted use, you will need to obtain permission directly from the copyright holder. To view a copy of this license, visit http://creativecommons.org/licenses/by/4.0/.

(C) The Author(s) 2017 